\title{
Étude des préconceptions de la voiture autonome en vue d'une éducation à la sécurité routière appropriée
}

A study of preconceptions of the autonomous car with a view to adequate road safety education

\section{Émeline Ah-tchine et Erica de Vries}

\section{OpenEdition Journals}

Édition électronique

URL : https://journals.openedition.org/educationdidactique/5162

DOI : 10.4000/educationdidactique.5162

ISSN : 2111-4838

Éditeur

Presses universitaires de Rennes

\section{Édition imprimée}

Date de publication : 30 décembre 2019

Pagination : 127-139

ISBN : 978-2-7535-8041-1

ISSN : 1956-3485

\section{Référence électronique}

Émeline Ah-tchine et Erica de Vries, «Étude des préconceptions de la voiture autonome en vue d'une éducation à la sécurité routière appropriée », Éducation et didactique [En ligne], 13-3 | 2019, mis en ligne le 03 janvier 2021, consulté le 10 octobre 2022. URL : http://journals.openedition.org/ educationdidactique/5162 ; DOI : https://doi.org/10.4000/educationdidactique.5162 


\title{
ÉTUDE DES PRÉCONCEPTIONS DE LA VOITURE AUTONOME EN VUE D'UNE ÉDUCATION À LA SÉCURITÉ ROUTIÈRE APPROPRIÉE
}

\author{
Émeline Ah-tchine \\ Laboratoire de Recherche sur les Apprentissages en Contexte, Univ. Grenoble Alpes \\ Erica de Vries \\ Laboratoire de Recherche sur les Apprentissages en Contexte, Univ. Grenoble Alpes
}

\begin{abstract}
La voiture autonome a été longtemps idéalisée dans la fiction et pourrait demain exister de manière concrète. Cet article propose une étude préalable à l'introduction de la voiture autonome dans la circulation nécessitant des adaptations dans le code de la route et dans l'éducation à la sécurité routière. Dans cette démarche, nous tenons compte du fait qu'il s'agit d'une innovation technologique qui n'existe pas encore sous sa forme finale dans la vie de tous les jours. Nous étudions le cas de la voiture autonome à travers trois volets qui s'appuient sur des domaines de connaissances en sciences de l'éducation avec leurs méthodologies associées : les questions socialement vives, les capacités projetées de la voiture autonome telles que relevées dans les savoirs savants, et les profils de préconceptions rencontrés dans la société à l'heure d'aujourd'hui. Létude empirique a permis de caractériser des profils de préconceptions qui permettront d'élaborer des préconisations pour une éducation à la sécurité routière.
\end{abstract}

Mots-clés : préconceptions, innovation, question socialement vive, éducation à la sécurité routière, voiture autonome.

\section{A study of preconceptions of the autonomous car with a view to adequate road safety education}

For a long time, autonomous cars have been idealized and fictional products which soon could come to reality. This article presents a study prior to the implementation of the autonomous car on public roads that will require updating road safety rules and road safety education. Our approach involves considering the autonomous car as a technological innovation, which is not yet available for the public. We examine the autonomous car in three different ways founded on scientific knowledge in educational science and its associated methods: Socially Acute Questions (SAQ), the expected capacities of the autonomous car according to the experts, and different profiles of preconceptions currently present in society. An empirical study revealed the presence of distinct preconception profiles. These profiles will allow elaborating recommendations for appropriate road safety training.

Keywords : preconceptions, innovation, socially acute questions, road safety education, autonomous car.

Cette étude a été réalisée dans le cadre d'une thèse financée par une allocation doctorale de recherche de l'Université Pierre-Mendès-France (aujourd'hui Univ. Grenoble Alpes) et en partenariat avec Ideas Laboratory. 


\section{INTRODUCTION}

La sécurité routière fait partie des sujets importants dans l'enseignement. D'après l'article D312-43 du code de l'éducation, les établissements du premier et du second degré doivent assurer un enseignement des règles de sécurité routière transdisciplinaire appelant à « l'acquisition des élèves de la connaissance des règles de sécurité et des comportements adaptés qui en découlent » (Article D312-44). Les enseignants disposent de tableaux pédagogiques qui rassemblent les savoirs et savoir-faire avec des propositions de situations pédagogiques et des grilles d'évaluation associées. Les écoles maternelles et élémentaires délivrent une attestation de première éducation à la route (APER) suivis par les attestations scolaires de sécurité routière (ASSR) en classe de $5^{\mathrm{e}}$ et $3^{\mathrm{e}}$ au collège. Néanmoins, de même que la santé, l'environnement, ou le développement durable, la sécurité routière ne relève pas de "savoirs académiques neutres, dissociés de tous contextes » (Lange $\&$ Victor, 2006). Bien qu'il existe un programme, des ressources pédagogiques, des modalités d'évaluation, et des attestations de réussite, on ne peut véritablement parler de référent académique pour l'éducation à la sécurité tel que pour les disciplines scientifiques. Plus particulièrement, l'éducation à la sécurité routière doit continuellement s'adapter à l'évolution de l'environnement routier (Courteaud $\&$ Roux, 2013) et aux modifications du code de la route qui s'ensuivent. De nos jours, cette adaptation nécessite d'anticiper l'introduction de nouvelles technologies telle que la voiture autonome (Comité interministériel de la sécurité routière, 2018). Dans le contexte d'une protection renforcée des piétons (voir le décret $n^{\circ} 2018-795$, section VI, article 13), la question des connaissances indispensables pour interagir avec le véhicule autonome se pose pour tous les usagers de la route, pour les enfants comme pour les adultes. Or, les savoirs sur le véhicule autonome ne sont ni partagés dans la société, ni même stabilisés parmi les experts.

La voiture autonome est un moyen de transport qui s'affranchit de l'intervention humaine pendant la conduite, ses capacités sont modulées selon le niveau d'autonomie correspondant. En remplacement de la supervision humaine, la voiture autonome est dotée de nombreux capteurs (radar, lidar, accéléromètre, gyromètre...) qui fournissent des informations sur l'environnement et l'état du trafic. Les données sont traitées par l'intelligence artificielle pour aboutir à une prise de décision en adéquation à une situation donnée. L'Organisation Internationale des Constructeurs d'Automobiles (OICA) propose une classification officielle de l'automatisation des voitures allant du niveau 1 , le conducteur est en total contrôle du véhicule, au niveau 5 , le véhicule est en totale autonomie sans aucune intervention humaine même en cas de problème. Ainsi, l'autonomie des voitures se fait de manière graduelle, c'est-àdire qu'une même voiture peut être à la fois conduite de manière traditionnelle avec chauffeur ou passer en mode automatique. Par conséquent, la convention de Vienne de 1968 exprimant la nécessité d'être maître de son véhicule a été récemment revue par la commission économique pour l'Europe des nations unies (UNECE). En effet, au regard des législations, tous les conducteurs doivent avoir reçu une formation certifiée au code de la route et à la conduite. Les particularités de la voiture autonome exigent ainsi une reconsidération du partage des responsabilités au niveau légal et par conséquent impliqueront tous les usagers de l'environnement routier.

Cet article propose une étude préalable à l'introduction de la voiture autonome dans la circulation et dans l'éducation à la sécurité routière. Dans cette démarche, nous devons tenir compte du fait qu'il s'agit d'une innovation technologique qui n'existe pas encore sous sa forme finale dans la vie de tous les jours. En effet, si la voiture autonome existe de manière conceptuelle, elle est peu tangible pour le grand public. Nous étudions le cas de la voiture autonome à travers trois volets qui s'appuient sur des domaines de connaissances en sciences de l'éducation avec leurs méthodologies associées : les questions socialement vives, les capacités projetées de la voiture autonome telles que relevées dans les savoirs savants, et les profils de préconceptions rencontrés dans la société à l'heure d'aujourd'hui.

\section{LA VOITURE AUTONOME : UNE QUESTION SOCIALEMENT VIVE?}

Les innovations technologiques font partie d'un champ disciplinaire où le savoir savant est en construction et n'a pas encore pu donner lieu au savoir enseigné (Alpe \& Barthes, 2013). Ce fut le cas pour l'informatique lorsqu'elle n'était pas encore identifiée comme discipline scolaire et les savoirs 
essentiels à sa compréhension pas encore bien définis (Baron \& Bruillard, 2001). Ainsi, la transposition didactique est moins aisée que pour des savoirs davantage établis (Chevallard, 1986). Or, dans le cas de la voiture autonome, on ne peut attendre la maturation des savoirs technologiques pour penser l'éducation. La formation de différents publics est nécessaire avant même l'émergence d'un consensus sur les phénomènes parmi les scientifiques et ingénieurs. Pour les nanotechnologies par exemple, la formation aux risques des nanoparticules sur le site Nanosmile (INSTN, n.d.) a été concomitante à la définition de ce qu'est une nanoparticule. Legardez et Simonneaux (2006) parlent de questions socialement vives (QSV) ou même de questions scientifiquement socialement vives (QSSV) pour aborder ces problématiques spécifiques de contenus d'enseignement qui ne sont pas stabilisés et pour lesquelles se posent de nombreuses questions. Pour la voiture autonome, par exemple, est-ce qu'elle serait plus sûre qu'une voiture conduite par un humain? Est-ce qu'elle s'affranchira des règles du code de la route lorsqu'une vie humaine est en jeu? Ou encore à qui donnerat-elle la priorité lorsque la situation mettra en jeu la vie à la fois des passagers de la voiture et des piétons extérieurs à la voiture?

Dans certaines disciplines scolaires, les objets d'éducation sont considérés comme concepts sociaux non stabilisés (Lange \& Victor, 2006) ou des questions socialement vives (Simonneaux, 2003). Pour Legardez (2006), on peut considérer une question socialement vive de trois façons. Premièrement, elle peut être vive dans la société. En effet, l'introduction de la voiture autonome dans la circulation est considérée comme un enjeu par la société. Dans un traitement médiatique récent, on s'interroge sur des questions d'éthique et de responsabilité en cas d'accident. Ainsi, une bonne partie des citoyens, dont les acteurs scolaires, ont connaissance de la question. Ensuite, elle peut être vive dans les savoirs de référence à travers des débats entre spécialistes des champs disciplinaires. Pour la voiture autonome, des controverses existent surtout sur ce qu'elle sera capable de faire et sur le degré de ressemblance d'une voiture autonome à une voiture traditionnelle conduite par un humain. Les limites et portées de l'intelligence artificielle en général sont au centre de ces débats. Enfin, toujours selon Legardez (2006), la question pourrait être vive dans les savoirs scolaires. Pour la voiture autonome, l'existence d'exemples déjà mythiques, tels que les voitures Google et Tesla, des documents abondants accessibles sur internet, des vidéos notamment, font que les élèves peuvent y être directement confrontés et sont ainsi susceptibles d'interroger leurs enseignants.

En effet, l'implémentation d'une technologie non aboutie ne pourra se faire sans une éducation aux nouveaux risques pour tous les usagers. Ainsi, comme l'indique Simonneaux (2003), face à des technosciences controversées, avec des savoirs socialement construits, il ne s'agit pas d'une transposition didactique de savoirs savants, mais d'un questionnement des connaissances et savoirs de base, qui comme les objets mêmes, sont soumis à des modifications dues aux innovations technologiques. Ils représentent un défi en ce que la conception d'une « éducation à... » requière de l'anticipation.

\section{SELON LES SPÉCIALISTES, DE QUEL TYPE D'ENGIN S'AGIT-IL?}

La voiture autonome évoque l'imaginaire autour des robots et de l'intelligence artificielle. L'intrusion de l'intelligence artificielle dans tous les domaines interroge aujourd'hui comme en témoignent de multiples dossiers dans la presse (par exemple « $\mathrm{Au}$ coeur de l'IA » dans Le Monde). La voiture autonome n'est pas conduite par une personne physique et humaine, le contrôle est sous l'égide d'un programme exécuté par une machine et s'appuie ainsi sur l'intelligence artificielle. Or, les avis sur les capacités réelles, actuelles ou futures, de ces objets du futur divergent même au sein de la communauté des experts (Hutson, 2017; Lawrence, 2017). Plus particulièrement, ces capacités sont implicitement ou explicitement comparées aux capacités d'un conducteur humain au volant d'une voiture traditionnelle. Une classification d'objets innovants n'est pas disponible aujourd'hui. En guise d'analyse a priori des savoirs savants, nous posons cette question des différentes capacités avérées, supposées, ou projetés de la voiture autonome.

Aujourd'hui, il existe des prototypes fonctionnels de la voiture autonome grâce aux nombreux développeurs qui font la course au premier prototype viable (Google car, Mercedes F015, Navya Arma...). Hussain et Zeadally (2018), dans une récente revue, pointent les dernières avancées scientifiques et technologiques de la voiture autonome. Sur le plan tech- 
nique, on distingue différentes capacités nécessaires pour la conduite en autonomie : la perception, la localisation, la planification, et le contrôle (Jo et al., 2014; Levinson et al., 2011). Des capteurs (lidars, radars, GPS, accéléromètres, gyroscopes, etc.), des modules de traitement de l'information, et des effecteurs gèrent l'état de l'environnement proche et lointain, la navigation, la planification d'itinéraire, et le contrôle des manœuvres. Cependant, des avancées techniques sont nécessaires afin d'améliorer la finesse et la précision. Les capacités techniques incluent également la partie prise de décision qui s'appuie sur l'intelligence artificielle. La difficulté réside dans la programmation des algorithmes pour appréhender et prédire toutes les situations possibles et y associer une prise de décision adaptée, éthique et appropriée. De nombreuses recherches sont effectuées pour améliorer les performances de la voiture autonome et son implémentation chez le grand public (Bimbraw, 2015; Fagnant \& Kockelman, 2015; Hashimoto, Gu, Hsu, Iryo-Asano, \& Kamijo, 2016; Jian, Lizhong, \& Daoliang, 2005; Jo, Kim, Kim, Jang, \& Sunwoo, 2015; Millard-Ball, 2016; Payre, Cestac, \& Delhomme, 2014). Hussain et Zeadally (2018) répertorient également les défis pour l'avenir. Outre sur le plan technique, les défis se situent sur le plan non-technique (confiance des consommateurs, coût, et les réactions du public par rapport aux accidents survenus et médiatisés récemment), sur le plan de la réglementation (quelles règles en cas d'accident impliquant un choix éthique (Coca-Vila, 2018; Fleetwood, 2017)), et sur le plan social (l'acceptabilité aux yeux du grand public d'une voiture pouvant sacrifier ses passagers pour le bien-être de la société (Bonnefon, Shariff, \& Rahwan, 2016)). Sur tous ces plans, de nombreuses imprécisions restent en suspens remettant en cause la fiabilité et la faisabilité de l'engin.

Comme l'objet technologique n'existe pas encore sous sa forme finale, il est difficile de prévoir ses capacités et par conséquent les problèmes soulevés lors de son implémentation. Il s'agit ainsi de connaître et de tenir compte des capacités potentielles, au vu des progrès en intelligence artificielle, des différents modules, presque boîtes noires, qui permettront la ressemblance de la voiture autonome à une voiture traditionnelle avec un conducteur humain. Les informaticiens distinguent en effet différents paliers de fonctionnement intelligent de la part des machines :
- Machines réactives. Le type 1 , selon Hintze (2016), consiste en des machines réactives. Comme Deep Blue, le superordinateur IBM qui pouvait jouer aux échecs, à ce niveau, le système ne peut ni mémoriser, ni utiliser ses expériences pour prendre des décisions informées. En quelque sorte, en termes de la nature de l'intelligence, la machine n'est pas plus évoluée qu'une machine à laver avec des programmes de lavage préprogrammés ou un robot d'usine qui exécute des programmes ou qui peut éviter des obstacles.

- Mémoire limitée. Des machines de type 2 possèdent en plus des capacités limitées de mémoire. Hintz considère la voiture autonome de ce deuxième type. Elles captent la vitesse et la trajectoire d'autres voitures, les combinent avec des connaissances sur le marquage au sol, les feux de circulation, pour se diriger. Selon ce type, ces voitures peuvent s'appuyer sur des algorithmes sophistiqués d'apprentissage machine (deep learning) pour identifier des objets. Par exemple, estce qu'un objet mouvant identifié est un autre véhicule, un piéton, une bicyclette, un animal? Alimenté par de nombreuses images, un tel algorithme examine une scène et devine le type d'objet. La plupart de ses suppositions initiales seront fausses et l'algorithme modifie les paramètres internes ou des parties de sa structure en fonction des erreurs initiales et essaie à nouveau. Ce processus se poursuit et consiste à rejeter les modifications qui réduisent la précision de l'algorithme et à conserver les modifications qui augmentent la précision jusqu'à classer correctement toutes les images. Ensuite, de nouvelles images sont présentées à l'algorithme qui les classera avec une grande précision. À ce moment-là, on dit que l'algorithme a « appris ». Le récent accident mortel entre un piéton et une voiture autonome à Tempe en Arizona, est un exemple de l'échec de l'algorithme n'ayant pas su correctement identifier le piéton présent sur la chaussée.

- Théorie de l'esprit. Les machines de type 3 intègrent une théorie de l'esprit. En effet, un conducteur humain observe et raisonne sur le comportement d'autres humains. Au centre du débat aujourd'hui est de savoir s'il est possible de construire une machine qui peut s'appuyer sur le comportement d'autrui et selon les experts, les machines d'aujourd'hui n'en sont pas capables. La part de la conduite qui 
concerne l'interprétation des comportements des autres utilisateurs de la route et la communication n'est pas intégrée. Ainsi, si l'on admet que les algorithmes « apprennent » dans une certaine mesure (type 2), la voiture autonome ne peut apprendre à partir de toutes les expériences comme le font les humains.

- Conscience de soi. Enfin, des machines de type 4 seraient des machines possédant la réflexivité ou la conscience de soi. L'humain possède cette qualité en ce qu'il peut s'observer lui-même et raisonner sur ses propres états, processus, décisions et phénomènes. Il peut analyser qu'il a eu un comportement freiner, klaxonner, accélérer - parce qu'il a eu une émotion - peur, colère, frustration - et s'appuyer sur ces observations pour interpréter le comportement d'autrui à l'avenir.

La classification de Hintz a l'avantage de rendre la comparaison avec l'humain explicite en ce que l'intelligence artificielle sait faire et ne pas faire aujourd'hui. Des débats existent au sein des spécialistes à la fois au sujet des capacités nécessaires et suffisantes pour introduire la voiture autonome dans la circulation et au sujet des garanties de pouvoir produire ces capacités à l'avenir. Sur ce dernier sujet, certains pointent l'existence de différentes «familles » plus ou moins confiantes, sceptiques, ou même hostiles au développement des capacités des machines (Cassini, Piquard, \& Larousserie, 2017).

Les capacités attribuées aux objets pourront avoir des conséquences importantes pour les comportements adoptés. Par ailleurs, elles peuvent être à l'origine de malentendus entre différents acteurs. Dans le courant de recherche sur le changement conceptuel, ce type de problème a été théorisé comme un problème d'attribution divergente d'un concept à une catégorie d'objets. Plus précisément, le problème de l'attribution erronée d'un concept à une catégorie a été décrit par Chi, Slotta, \& de Leeuw (1994). Ces auteurs formalisent la compréhension de concepts par leur classification dans une arborescence de catégories ontologiques déterminés par trois suppositions épistémologique, métaphysique et psychologique. L'ontologie a trait aux catégories de l'existence, à l'idée que l'on se fait des différentes sortes de choses qui existent. La supposition épistémologique considère que l'on peut catégoriser les entités du monde qui nous entoure suivant trois catégories ontologiques primaires : la matière, les processus et les états mentaux (voir figure 1). Ces catégories se décomposent par souscatégories hiérarchiques définies par des attributs ontologiques tels que « a lieu » pour la catégorie processus, ou encore "peut se réparer » pour la catégorie artéfact. Ainsi, les catégories d'un arbre ontologique se différencient par le fait qu'elles ne partagent pas les mêmes attributs ontologiques. Selon Chi, Slotta \& de Leeuw (1994), certaines difficultés dans l'apprentissage proviennent de l'incompatibilité entre la catégorie ontologique adoptée par les élèves et celle à laquelle un certain concept appartient pour les scientifiques. En effet, leurs travaux focalisent sur l'apprentissage des sciences. Les concepts scientifiques appartiennent souvent à la catégorie "interaction contrainte » dans la branche « processus ». Les conceptions alternatives, initiales, naïves ou erronées attribuent souvent ces concepts, comme la lumière, la chaleur, l'électricité, ou la force, à la catégorie "liquide " ou « solide " dans la branche "matière ». La compréhension d'un concept n'est possible que si l'individu "subi" une modification conceptuelle, un changement de catégorie. Cette modification peut être assez soudaine, du type « aha erlebnis» (voir aussi Lautrey, 2008).

L'arborescence ontologique présentée n'est qu'un exemple et ses catégories ne sont pas exhaustives. Elles sont heuristiques dans notre compréhension des difficultés dans les apprentissages de la physique. Nous proposons de théoriser les potentiels malentendus au sujet d'objets innovants, comme la voiture autonome, par le développement de la catégorie « artéfact » (ellipses blanches dans la figure 1) en nous fondant sur la typologie de Hintz. Cette catégorie "artéfact », délaissée par manque de pertinence pour l'apprentissage de la physique, se trouve au centre de la problématique des objets dits « intelligents ». Premièrement, on peut donc diviser les artéfacts en objets traditionnels sans intelligence artificielle - lampe, porte, stylo - et les objets programmés divisés eux-mêmes en quatre catégories selon les quatre types de Hintz.

Notons que cette proposition peut être à l'origine de débats. Par exemple, une machine à laver, même avec des programmes préréglés, peut être classée dans la catégorie "sans IA ». Or, aujourd'hui les fabricants vantent les mérites des lave-linge intelligents qui, équipés de capteurs, ajustent automatiquement la consommation d'eau, la température de lavage, la vitesse d'essorage, la durée du cycle et le nombre de rinçages, en fonction du poids du 
Figure 1 : L'arborescence ontologique étendue intégrant le développement de la catégorie « artéfact »

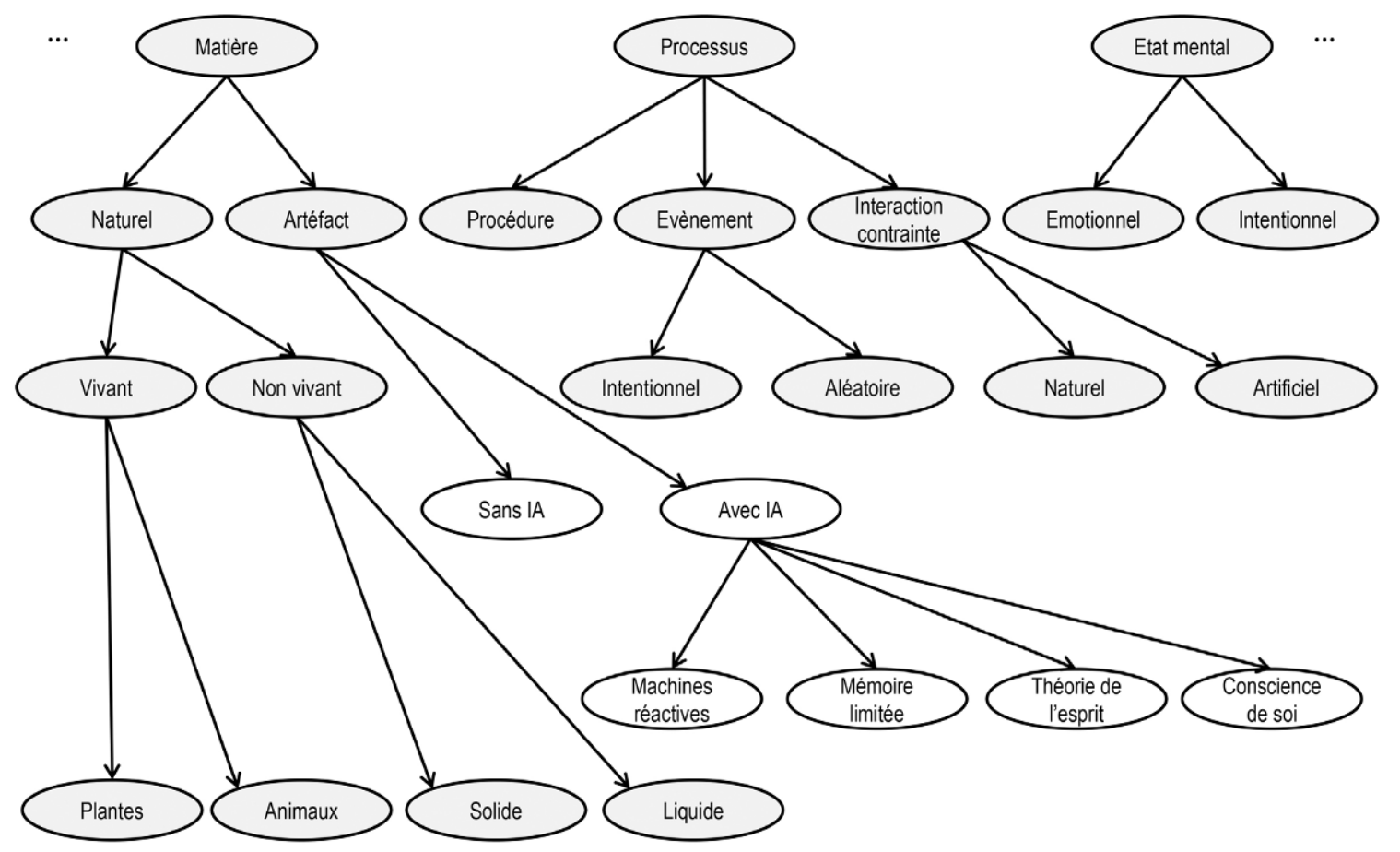

Les ellipses grisées figurent dans l'original de Chi, Slotta et de Leeuw (1994), les ellipses blanches constituent notre ajout.

linge et de son degré de salissure. Les machines à laver pourraient ainsi être classées comme intelligentes au premier palier, celui des « machines réactives ». Cet exemple montre le décalage entre les savoirs savants chez les ingénieurs et constructeurs et l'état des connaissances chez le grand public. Notre proposition d'une arborescence ontologique étendue est une première approximation pour pouvoir explorer les préconceptions potentielles de la voiture autonome.

\section{QUELLES PRÉCONCEPTIONS DE LA VOITURE AUTONOME?}

Pour la voiture autonome, ou le prototype le plus achevé, il n'existe pour l'instant aucun objet du quotidien avec des capacités comparables opérant dans un environnement ouvert et non contraint. Cependant, on pourrait s'appuyer sur des objets dont le fonctionnement est similaire comme les lignes de métro automatiques ou les robots ménagers, par exemple un robot de piscine. Or, la voiture autonome a un référent connu : la voiture traditionnelle. La différence majeure entre les deux est l'absence de chauffeur humain à qui on peut attribuer le contrôle de la voiture. En effet, dans la pratique, la communication entre le chauffeur humain et le piéton est un élément important pour la sécurité, par exemple dans la décision de traverser. On peut se poser la question des effets et des conséquences de l'absence de chauffeur (Brémond et al., 2012; Kaparias et al., 2012). Une traversée s'effectue selon des indices: une voiture qui décélère et un conducteur qui établit un contact visuel, qui acquiesce la présence d'un piéton, sont des indicateurs de moindre risque de collision. La voiture traditionnelle avec chauffeur est ainsi la réfé- 
rence et influencera la conception initiale qu'ont les piétons de la voiture autonome.

Le cas de la voiture autonome s'apparente ainsi à l'apprentissage de la physique lorsqu'il y a une similarité, mais en même temps une différence essentielle, entre deux concepts. Vosniadou $\&$ Brewer (1994) considèrent que les conceptions se construisent à partir de présuppositions innées ou acquises par la culture et les observations du monde extérieur. Ainsi, Vosniadou et Brewer considèrent le changement conceptuel comme un processus graduel de révision, de suppression, d'addition ou de suspension de ces présuppositions, modèles mentaux et croyances. Par exemple, Vosniadou \& Brewer (1992) répertorient différents modèles mentaux de la forme de la terre chez des enfants en école élémentaire (voir aussi Lautrey, 2008). Les élèves évoluent d'un modèle initial, une terre plate, vers un modèle scientifique, de type globe, en passant par des modèles synthétiques. Ces modèles synthétiques sont des tentatives de conciliation entre les présuppositions des élèves et le modèle reconnu scientifiquement. Ainsi, les auteurs ont pu découvrir des modèles de type globe creux avec un sol dur à l'intérieur; une combinaison de l'observation d'un monde plat avec la reconnaissance scientifique d'un monde globe sphérique. En résumé, les conceptions initiales, d'après ces travaux et ceux de Chi $(1994,2013)$ sont homogènes chez différentes catégories d'individus, robustes, c'est-à-dire que les individus y tiennent fermement, cohérentes à travers le temps et dans des situations variées, persistantes à travers les niveaux scolaires et les âges, systématiques en ce qu'elles forment une théorie cohérente, et récapitulatives de l'histoire des sciences.

L'objectif de l'étude empirique est d'explorer les préconceptions de la voiture autonome dans la société à l'heure actuelle. Selon les études des attentes des usagers (Kyriakidis, Happee, \& de Winter, 2015; Miller et al., 2016; Verberne, Ham, \& Midden, 2012), la question de la sécurité et de la confiance dans la voiture autonome pourrait être prédominante. Plus précisément, deux tonalités sont attendues caractérisées soit par la ressemblance à l'humain et ainsi une confiance dans le respect du code de la route, soit au contraire par la différence par rapport à l'humain et ainsi une méfiance des véhicules conduits par une machine. Nous explorons la présence de ces deux attitudes au moyen d'une analyse factorielle exploratoire sur une échelle sémantique différentielle soumise à des étudiants en licence de sciences humaines et sociales. Par ailleurs, nous explorons l'existence de profils distincts par le biais d'une analyse hiérarchique de nuées dynamiques sur le contenu des réponses à une question ouverte posée au même public. Nous aboutirons ainsi à un relevé des profils essentiellement différents présents dans la société. En effet, les caractéristiques des préconceptions initiales relevées dans la littérature (homogénéité, persistance) permettent de faire l'hypothèse que ces mêmes profils existeraient chez un public plus jeune.

\section{MÉTHODE}

Nous avons élaboré un questionnaire exploratoire composé de trois parties : une échelle sémantique différentielle, des questions sollicitant les préconceptions et une partie générale sur la démographie, avec notamment la connaissance du code de la route et l'obtention du permis de conduire.

\section{Échantillon}

180 étudiants de licence en sciences humaines et sociales d'une université française ont été recrutés pour l'étude. Cet échantillon se compose de $14 \%$ d'hommes pour une moyenne d'âge de 20 ans et un écart-type de 3,2 ans. 83,3\% des participants ont obtenu le code de la route et $74,4 \%$ ont obtenu le permis B.

\section{L'échelle sémantique différentielle}

L'échelle sémantique différentielle a pour but de mesurer les attitudes et a été développé par Osgood en 1964. Elle consiste en une échelle d'items avec à chaque extrémité des adjectifs contraires représentant des dimensionnalités du concept étudié en termes d'intensité et de direction (Johnson, 2012). Cet outil a été repris et utilisé pour connaître les attitudes sur les nouvelles technologies (Ajani \& Stork, 2013; Johnson, 2012). Pour la construction de notre échelle, nous avons choisi des adjectifs caractérisant les qualités d'un chauffeur humain et des caractéristiques technologiques d'une voiture, comme par exemple « respectueuse » et « connectée ». L'échelle sémantique différentielle proposée est constituée de 11 items de cinq points (Tableau 1 ). 
Tableau 1 : Échelle sémantique différentielle

$\begin{array}{rllllll}\text { Intelligente } & 1 & 2 & 3 & 4 & 5 & \text { Sans intelligence } \\ \text { Responsable } & 1 & 2 & 3 & 4 & 5 & \text { Irresponsable } \\ \text { Connectée } & 1 & 2 & 3 & 4 & 5 & \text { Déconnectée } \\ \text { Compétente } & 1 & 2 & 3 & 4 & 5 & \text { Incompétente } \\ \text { Informée } & 1 & 2 & 3 & 4 & 5 & \text { Ignorante } \\ \text { Respectueuse } & 1 & 2 & 3 & 4 & 5 & \text { Irrespectueuse } \\ \text { Inoffensive } & 1 & 2 & 3 & 4 & 5 & \text { Dangereuse } \\ \text { Rapide } & 1 & 2 & 3 & 4 & 5 & \text { Lente } \\ \text { Manuelle } & 1 & 2 & 3 & 4 & 5 & \text { Automatique } \\ \text { Machine } & 1 & 2 & 3 & 4 & 5 & \text { Humaine } \\ \text { Sensible } & 1 & 2 & 3 & 4 & 5 & \text { Insensible }\end{array}$

\section{L'invite pour solliciter les préconceptions}

Le questionnaire contenait deux questions pour solliciter les préconceptions. La première question est une question de contrôle pour déterminer l'expérience préalable avec la voiture autonome : «Je n'en n'ai jamais entendu parler », «J'ai une vague idée de ce que ça peut être », "Je sais de quoi il s'agit », " Je m'y intéresse », " J'en ai déjà vu/essayé ». La seconde question est ouverte et invite les participants à donner leur conception initiale de la voiture autonome.

«Dans cette étude, nous nous intéressons à la voiture autonome, cette nouvelle technologie pas encore sur le marché, va bientôt pouvoir être commercialisée et faire partie de notre quotidien. C'est justement le sujet de conversation du jour avec vos ami(e)s, l'inévitable question « mais qu'est-ce qu'une voiture autonome? » est alors posée. Vous vous proposez volontaire pour présenter la voiture autonome en quelques mots. Veuillez retranscrire ci-dessus vos explications. »

Cette question ouverte n'impose pas de cadre. Les participants, avec leurs propres mots, choisissent de mettre en valeur la nature de la voiture autonome, ses caractéristiques fonctionnelles, son usage ou encore des comparaisons possibles avec la voiture traditionnelle.

\section{Procédure}

Le questionnaire a été présenté et soumis à des étudiants en amphithéâtre. La participation volontaire et anonyme des étudiants s'est déroulée avant le début d'un cours avec l'autorisation de l'enseignant. La passation a duré 10 minutes au bout desquelles les questionnaires ont été récupérés et les participants remerciés.

\section{RÉSULTATS}

Dans notre échantillon, on observe $11.1 \%$ des participants indiquant n'avoir jamais entendu parler de la voiture autonome. Par contre $59.4 \%$ des participants ont indiqué en avoir une vague idée et $25.6 \%$ assurent savoir de quoi il s'agit, avec $3.9 \%$ démontrant un grand intérêt sur le sujet. Ces résultats nous confortent dans l'idée qu'il s'agit d'un public avec de véritables conceptions initiales.

\section{Analyse factorielle exploratoire}

L'analyse factorielle exploratoire (AFE) permet de découvrir la structure sous-jacente à un ensemble de variables. Le tableau 2 montre moyennes et écart types, ainsi que les résultats de l'analyse factorielle exploratoire sur l'échelle sémantique différentielle. L'indicateur du Kaiser-Meyer-Olkin (KMO) mesurant l'adéquation de l'échantillon et l'interdépendance des variables (pas trop de corrélation entre elles) témoigne d'une valeur acceptable, $\mathrm{KMO}=.76$.

Trois items - manuelle-automatique, machinehumaine, et sensible-insensible - ne distinguent pas suffisamment entre individus et sont exclus de l'analyse. Par rapport à ces items, les moyennes dans le tableau 2 montrent que la voiture autonome est considérée plutôt machine, automatique, et insensible.

Dans le cas de la voiture autonome, l'AFE réduit les 11 items de l'échelle sémantique différentielle à un couple de facteurs orthogonaux. Les deux facteurs ayant une valeur Eigen de plus de 1 (critère de Kaiser) expliquent plus de $53 \%$ de la variance dans les items. L'interprétation des deux facteurs sous-jacents aux attitudes doit s'appuyer sur les coefficients de saturation les plus élevés (en gras dans le tableau 2). 
Le facteur 1 est composé des items ayant trait au caractère responsable, inoffensif, respectueux, intelligent et compétent de la voiture autonome. Ce facteur 1 semble ainsi incarner une forme de bienveillance attribuée à l'objet, comme si le respect et la responsabilité que l'on attribue aux conducteurs humains pouvaient être programmés dans la voiture autonome.

Tableau 2 :

Analyse factorielle exploratoire après rotation varimax (coefficients de saturation supérieurs à 35 apparaissent en gras)

\begin{tabular}{|c|c|c|c|c|}
\hline Items & Moyenne & St. Dev. & Facteur 1 & Facteur 2 \\
\hline $\begin{array}{l}\text { Responsable - } \\
\text { Irresponsable }\end{array}$ & 2.87 & 1.08 & .73 & .20 \\
\hline $\begin{array}{l}\text { Inoffensive - } \\
\text { Dangereuse }\end{array}$ & 3.59 & .97 & .60 & .18 \\
\hline $\begin{array}{l}\text { Respectueuse - } \\
\text { Irrespectueuse }\end{array}$ & 2.64 & .92 & .59 & -.01 \\
\hline $\begin{array}{l}\text { Compétente - } \\
\text { Incompétente }\end{array}$ & 2.39 & .87 & .38 & .65 \\
\hline $\begin{array}{l}\text { Informée - } \\
\text { Ignorante }\end{array}$ & 2.24 & .98 & .25 & .54 \\
\hline $\begin{array}{l}\text { Intelligente - } \\
\text { Sans Intelligence }\end{array}$ & 1.98 & 1.11 & .43 & .52 \\
\hline $\begin{array}{l}\text { Connectée - } \\
\text { Déconnectée }\end{array}$ & 1.48 & .78 & .10 & .49 \\
\hline Rapide-Lente & 2.98 & .79 & -.07 & .29 \\
\hline $\begin{array}{l}\text { Manuelle - } \\
\text { Automatique }\end{array}$ & 4.53 & .78 & & \\
\hline $\begin{array}{l}\text { Machine - } \\
\text { Humaine }\end{array}$ & 1.34 & .77 & & \\
\hline $\begin{array}{l}\text { Sensible - } \\
\text { Insensible }\end{array}$ & 3.49 & 1.15 & & \\
\hline Eigenvalues & & & 2.97 & 1.29 \\
\hline$\%$ variance & & & 37.14 & 16.12 \\
\hline$\alpha$ & & & .75 & .71 \\
\hline
\end{tabular}

Le facteur 2 est lié au caractère informé, intelligent, connecté et compétent de la voiture autonome et exprime ainsi une forme de technicité. Le facteur 2 correspondrait à l'aspect robotique, technologique, et informatique attribué à l'objet, une vision technocentrée confiante en l'innovation. Pour discriminer entre ces deux facteurs, l'un semble attribuer implicitement certaines qualités humaines à la voiture autonome, l'autre focalise sur la prouesse technologique que constitue l'objet. Cette configuration en deux facteurs nuance l'existence des deux tonalités attendues dans les préconceptions. Chaque tonalité se présente comme une dimension en soi : une dimension positionne par rapport à une bienveillance quasi-humaine en prenant en compte la sécurité, l'autre positionne, malgré l'absence de l'humain, par rapport au degré de confiance à l'automaticité et à l'intelligence artificielle.

\section{Analyse hiérarchique de nuées dynamiques}

Une analyse hiérarchique de nuées dynamiques (Borgen \& Barnett, 1987; Yim \& Ramdeen, 2015) permet de regrouper des individus ayant des profils similaires à partir d'un ensemble de variables. Dans notre cas, les variables sont constituées d'éléments de contenu repérés dans les écrits des participants. Pour cela, les réponses à la question ouverte ont été codées suivant la méthode présentée par Chi (1997). Cette méthode procède par le découpage des réponses en unités élémentaires, puis l'identification des éléments de contenu à l'aide d'une grille d'analyse. La grille d'analyse finale (première colonne du tableau 3) contient treize éléments de contenu, tels que " exécute une tâche », identifiés dans les réponses. La méthode de Chi résulte en un relevé des éléments de contenu pour chaque participant constituant la base de données pour l'analyse.

L'analyse hiérarchique de nuées dynamiques selon la méthode de Ward résulte en cinq clusters (profils). Trois éléments de contenu - reconnaissance signalisation, robotique et dangereux - avaient un nombre d'occurrences trop faible $(<4 \%)$ pour être inclus dans l'analyse. Le tableau 3 montre, pour chaque élément de contenu, le pourcentage de participants l'ayant mentionné dans chaque cluster et au total. Par exemple, 50 pourcent des individus dans le Cluster 1 mentionnent " Conduite seule », tandis qu'aucun $(0 \%)$ dans le même cluster ne mentionne «Exécute une tâche ».

Pour interpréter les résultats de cette analyse, nous devons regarder les pourcentages les plus élevés d'abord dans chaque ligne pour décrire la distribution des éléments de contenu dans l'échantillon, et ensuite dans chaque colonne pour caractériser les clusters/profils. En premier lieu, concernant la distribution, certains éléments de contenu montrent un pourcentage relativement élevé pour tous les clusters et ainsi ne permettent pas de distinguer entre profils. Par exemple, la propriété "conduite seule" de 
la voiture autonome est mentionnée en moyenne par 44 pourcent des participants et par plus d'un tiers des participants dans tous les clusters. D'autres éléments de contenu, comme par exemple la propriété « Écologique/Propre », n'est mentionnée que par 5 pourcent des participants qui se retrouvent majoritairement dans le Cluster 4.

En deuxième lieu, concernant la caractérisation des profils, nous avons attribué un label à chacun des profils en nous fondant sur les pourcentages supérieurs à $19 \%$ (en gras dans le tableau 3). Il s'agit de comprendre le focus de la conception de la voiture autonome pour chaque cluster/profil à partir des éléments de contenu.

- Sécurité. Dans le cluster 1 qui rassemble le plus d'effectifs, les participants considèrent la voiture autonome sécurisée, le chauffeur inutile et soulignent la présence de capteurs. Ainsi, dans ce profil de préconception, l'humain aux commandes du véhicule est considéré comme superflu car la voiture autonome ne présenterait pas de danger du fait de ses nombreux capteurs, des dispositifs technologiques qui détectent toutes informations nécessaires. - Définition. Le deuxième cluster ne semble caractérisé que par une définition sémantique simple sans conception particulière de la voiture autonome. En effet, les éléments de contenu ne font que reprendre les termes directement liés à la voiture autonome « chauffeur inutile » et « autonome ». Ce profil suggère des conceptions très primaires sans réflexion plus poussée sur l'objet.

- Fonction. Le troisième cluster se concentre sur les fonctionnalités de la voiture autonome en détaillant la nécessité d'un paramétrage. Il est caractérisé par les éléments de contenu " chauffeur inutile », " exécute une tâche », « réglages nécessaires » et « connectée et intelligente ». Autrement dit, dans ce profil de préconceptions, les individus sont bien avertis de ce qui pourrait remplacer le chauffeur humain grâce notamment à l'intelligence de la voiture et à sa connectivité. Il suffirait de faire des réglages pour pouvoir accomplir la mission de transport.

- Intelligence. Le quatrième cluster rend compte des éléments de contenu « connectée et intelligente » mais également " propre et écologique " avec à nouveau l'inutilité d'un chauffeur aux commandes. Ce quatrième profil suggère une préconception qui ne prend pas en compte la (non) sécurité présupposée de ce type de voiture, mais focalise plutôt sur le caractère intelligent, réfléchi et responsable de la voiture en matière de pollution et de gestion du trajet par exemple.

Tableau 3 : Pourcentage de participants dans chaque cluster par élément de contenu

\begin{tabular}{|c|c|c|c|c|c|c|}
\hline Élément de contenu & Cluster 1 & Cluster 2 & Cluster 3 & Cluster 4 & Cluster 5 & Total \\
\hline Conduite seule & 50.0 & 44.4 & 36.6 & 46.2 & 43.5 & 44.4 \\
\hline Autonome & 11.1 & 38.9 & 12.2 & 15.4 & 100.0 & 28.9 \\
\hline Chauffeur inutile & 27.8 & 100.0 & 46.3 & 42.3 & 4.3 & 45.6 \\
\hline Chauffeur utile & 7.4 & & 2.4 & & 21.7 & 5.6 \\
\hline Exécute une tâche & & & 75.6 & 11.5 & 17.4 & 21.1 \\
\hline Réglages nécessaires & 3.7 & & 39.0 & 3.8 & 4.3 & 11.1 \\
\hline Sécurité & 31.5 & 5.6 & 17.1 & 15.4 & 8.7 & 17.8 \\
\hline Connectée/Intelligente & 11.1 & & 19.5 & 96.2 & & 21.7 \\
\hline Capteurs & 70.4 & & 7.3 & 3.8 & 26.1 & 26.7 \\
\hline Écologique/Propre & & 5.6 & 2.4 & 19.2 & 4.3 & 5.0 \\
\hline Reconnaissance signalisation & & & & & & $<4.0$ \\
\hline Robotique & & & & & & $<4.0$ \\
\hline Dangereux & & & & & & $<4.0$ \\
\hline Total & 30.0 & 20.0 & 22.8 & 14.4 & 12.8 & 100.0 \\
\hline Label attribué au profil & Sécurité & Définition & Fonction & Intelligence & Chauffeur & \\
\hline
\end{tabular}


- Chauffeur. Le dernier cluster traduit un profil de conception avec les éléments de contenu « autonome », " chauffeur utile » et « capteurs ». Ce profil de préconception de la voiture autonome invoque les caractéristiques des voitures équipées de dispositifs d'aide à la conduite qui sont aujourd'hui déjà présents sur le marché.

Ainsi, l'analyse a permis de montrer l'existence de profils distincts de préconceptions au sujet de la nature de la voiture autonome. Ces profils font écho à différents degrés de ce que l'on peut entendre dans les médias. Par exemple, ils intègrent les souhaits d'innovation d'une voiture du futur : celle-ci devra être propre, programmable et intelligente et permettre à tous les usagers de la route d'être en sécurité.

On peut se poser la question de la relation entre ces cinq profils et les deux facteurs de bienveillance et de technicité trouvés dans la première analyse. Pour ce faire, nous calculons la moyenne des scores pour les facteurs pour chaque profil. Les deux profils Sécurité et Chauffeur se ressemblent en étant positifs sur les deux facteurs $(.13, .15$ et $.06, .07$ respectivement). Ces deux profils réunissent ainsi à la fois la bienveillance humaine et la technicité. Le profil Définition présente des moyennes modérément négatives sur les deux facteurs $(-.12,-.08)$ ne soulignant ainsi ni qualité humaine, ni prouesse technique. Le profil Fonction présente une moyenne modérément négative sur le deuxième facteur $(.05,-.13)$, et enfin le profil Intelligence présente une moyenne négative sur le premier facteur $(-.23,-.03)$. Il semblerait bien qu'il y ait deux tonalités dans les attitudes et dans les profils.

\section{DISCUSSION}

La voiture autonome n'est pas présente dans notre quotidien mais en tant qu'une des innovations les plus attendues, elle fait partie d'une réalité proche et risque d'exiger des adaptations dans le code de la route et l'éducation à la sécurité routière. En effet, l'intelligence artificielle est utilisée de plus en plus et bouscule nos catégories et nos conceptions. L'étude a permis de mettre en lumière les éléments caractérisant les préconceptions sur la voiture autonome. Deux facteurs de bienveillance et de technicité dans les attitudes ont été mis en évidence. Le premier facteur faisant état de valeurs humaines tels que le respect, la responsabilité, et le danger explique le plus de variance. Le deuxième facteur fait le rapport d'une voiture très technique renseignée par des capteurs. Par ailleurs, cinq profils distincts ont été établis avec des accents différents et à des niveaux fluctuants de détail. Le profil Définition, par exemple, présente une préconception limitée à la définition sémantique de l'objet et est ainsi une conception initiale à faible niveau de connaissance. Comme pour le profil Intelligence qui omet l'aspect sécurité de la voiture autonome, ces profils requièrent une formation plus poussée sur la voiture autonome et son rôle concernant tous les usagers de l'environnement routier.

À travers la littérature, nous avons mis en évidence quatre catégories ontologiques potentielles pour l'utilisation de l'intelligence artificielle. Quelle serait la relation avec les cinq profils de préconceptions identifiés dans l'étude empirique? Les participants au profil Définition, par exemple, restent au niveau artéfact dans l'arbre ontologique et ne semblent pas posséder ni sous-catégories avec et sans intelligence artificielle, ni différents types d'intelligence artificielle. Le profil Fonction avoisine la catégorie machines réactives en ce que les participants témoignent, comme pour la machine à laver, d'intégration de quelques capacités programmables. À l'autre extrême du spectre des différents types d'intelligence artificielle, les participants au profil Sécurité semblent intégrer toutes les précautions que pourraient prendre un chauffeur humain et ainsi relever de la catégorie conscience de soi.

Une limitation de notre étude tient à la population interrogée issue majoritairement d'étudiants en sciences humaines et sociales et comportant un faible pourcentage d'hommes. Si ce panel a permis un aperçu généraliste des profils présents dans la société aujourd'hui, les réponses à la question ouverte ne contenaient pas de détails techniques. Ainsi, des travaux futurs pourraient porter sur les caractéristiques des attitudes et profils chez d'autres publics, en particulier des étudiants dans des formations scientifiques et techniques. Par ailleurs, l'ingénierie pédagogique suivant nos résultats impliquera de vérifier l'existence de ces profils de préconceptions et leurs fréquences chez des enfants scolarisés de l'école primaire au lycée.

Notre démarche ouvre la voie vers l'ingénierie pédagogique d'une éducation à la sécurité routière anticipée et adaptée aux objets auparavant non catégorisables. Il s'agirait d'élaborer des programmes 
appropriés quant aux différentes catégories ontologiques et profils de préconceptions. Or, le problème est que les catégories sont inexistantes et non développées même chez le grand public. En amont des préconisations sur les comportements à adapter, il s'agit d'enseigner sur l'existence même de catégories différentes d'artefacts due à l'intelligence artificielle. À l'inverse, ceux qui ont des catégories de l'intelligence artificielle, ou qui peuvent considérer des machines comme étant intelligentes, paraissent avoir une vision trop optimiste des capacités de la voiture autonome. Il s'agirait alors de faire prendre conscience de ce que peuvent réaliser les algorithmes. Ainsi cela dépendra de la levée des secrets industriels sur les algorithmes.

La meilleure remise en cause de ses propres conceptions se fonde sur une interaction fréquente avec l'objet. Or, pour la voiture autonome, une exposition répétée et des expériences multiples et variées ne peuvent être envisagées pour des raisons de sécurité. Notre démarche contribue à l'identification des conceptions erronées sans exposer au danger. Ainsi, la prise en compte des préconceptions de la voiture autonome peut s'avérer un élément important dans la prévention à la sécurité routière. Si la démarche est indispensable en raison de la particularité de la voiture autonome, à la fois semblable à la voiture traditionnelle et représentant une véritable innovation, nous pensons qu'elle pourra porter ses fruits pour d'autres objets technologiques novateurs à l'avenir.

\section{RÉFÉRENCES}

Ajani, T., \& Stork, E. (2013). Creating a Semantic Differential Scale for measuring users' perceptions and attitudes toward emerging technologies. In 2013 Proceedings of the Conference for Information Systems Applied Research ISSN (pp. 2167-1508) [http://proc. conisar.org/2013/pdf/2564.pdf] (PDF).

Alpe, Y., \& Barthes, A. (2013). De la question socialement vive à l'objet d'enseignement : comment légitimer des savoirs incertains? Les dossiers des sciences de l'éducation, (29), 33-44 [https://doi.org/10.4000/dse.95].

Baron, G.-L., \& Bruillard, E. (2001). Une didactique de l'informatique? Revue Française de Pédagogie, 163-172.

Bimbraw, K. (2015). Autonomous Cars: Past, Present and Future - A Review of the Developments in the Last Century, the Present Scenario and the Expected Future of Autonomous Vehicle Technology: In Proceedings of the 12th International Conference on Informatics in Control, Automation and Robotics (pp.
191-198). Colmar, Alsace, France: SCITEPRESS Science and and Technology Publications [https://doi. org/10.5220/0005540501910198].

Bonnefon, J.-F., Shariff, A., \& Rahwan, I. (2016). The social dilemma of autonomous vehicles. Science, 352(6293), 1573-1576.

Borgen, F. H., \& Barnett, D. C. (1987). Applying cluster analysis in counseling psychology research. Journal of Counseling Psychology, 34(4), 456.

Brémond, R., Tom, A., Désiré, L., Gigout, E., Granié, M.-A., \& Auberlet, J.-M. (2012). Visual cues in pedestrian's crossing decision: in search of a quantitative model. In Proc. Transportation Research Board Annual Meeting, Washington DC [http://perso.lcpc.fr/roland.bremond/ documents/TRB12_Bremond.pdf] (PDF).

Cassini, S., Piquard, A., \& Larousserie, D. (2017, December 31). Les 5 familles de l'intelligence artificielle. Le Monde.fr [http://www.lemonde.fr/pixels/ article/2017/12/31/les-5-familles-de-l-intelligence-artificielle_5236310_4408996.html].

Chevallard, Y. (1986). La Transposition didactique: du savoir savant au savoir enseigné. (Recherches en didactique des mathématiques). JSTOR [http://www.jstor.org/ stable/41162650].

Chi, M. T. H. (1997). Quantifying qualitative analyses of verbal data: A practical guide. The Journal of the Learning Sciences, 6(3), 271-315.

Chi, M. T. H., Slotta, J. D., \& de Leeuw, N. (1994). From things to processes: a theory of conceptual change for learning science concepts. Learning and Instruction, 4, 27-43.

Coca-Vila, I. (2018). Self-driving cars in dilemmatic situations: An approach based on the theory of justification in criminal law. Criminal Law and Philosophy, 12(1), 59-82.

Comité interministériel de la sécurité routière. (2018). Sauvons plus de vies sur nos routes (Dossier de presse). Comité interministériel de la sécurité routière. Retrieved from www.gouvernement.fr/partage/9885comite-interministeriel-de-la-securite-routiere

Courteaud, L., \& Roux, C. (2013). Thème, histoire et problématique en éducation à la sécurité routière. Questions Vives. Recherches en éducation, (vol. 9, n¹9), 141-157 [https://doi.org/10.4000/questionsvives.1300].

Fagnant, D. J., \& Kockelman, K. (2015). Preparing a nation for autonomous vehicles: opportunities, barriers and policy recommendations. Transportation Research Part A: Policy and Practice, 77, 167-181.

Fleetwood, J. (2017). Public health, ethics, and autonomous vehicles. American Journal of Public Health, 107(4), 532-537.

Hashimoto, Y., Gu, Y., Hsu, L.-T., Iryo-Asano, M., \& Kamijo, S. (2016). A probabilistic model of pedestrian crossing behavior at signalized intersections for connected vehicles. Transportation Research Part C: Emerging Technologies, 71, 164-181 [https://doi. org/10.1016/j.trc.2016.07.011]. 
Hintze, A. (2016). Understanding the four types of $\mathrm{AI}$, from reactive robots to self-aware beings. The Conversation [http://theconversation.com/understanding-the-four-types-of-ai-from-reactive-robots-to-selfaware-beings-67616].

Hussain, R., \& Zeadally, S. (2018). Autonomous Cars: Research Results, Issues and Future Challenges. IEEE Communications Surveys \& Tutorials, 1-1 [https://doi. org/10.1109/COMST.2018.2869360].

Hutson, M. (2017). AI Glossary: Artificial intelligence, in so many words. American Association for the Advancement of Science.

INSTN. (n.d.). NANOSMILE - Accueil. (F. Tardif, Ed.). CEA [http://www.nanosmile.org/index.php/fr/].

Jian, L., Lizhong, Y., \& Daoliang, Z. (2005). Simulation of bi-direction pedestrian movement in corridor. PhysicaA: Statistical Mechanics and Its Applications, 354, 619-628 [https://doi.org/10.1016/j.physa.2005.03.007].

Jo, K., Kim, J., Kim, D., Jang, C., \& Sunwoo, M. (2014). Development of Autonomous Car Part I: Distributed System Architecture and Development Process. IEEE Transactions on Industrial Electronics, 61(12), 71317140 [https://doi.org/10.1109/TIE.2014.2321342].

Jo, K., Kim, J., Kim, D., Jang, C., \& Sunwoo, M. (2015). Development of Autonomous Car-Part II: A Case Study on the Implementation of an Autonomous Driving System Based on Distributed Architecture. IEEE Transactions on Industrial Electronics, 62 (8), 51195132 [https://doi.org/10.1109/TIE.2015.2410258].

Johnson, F. C. (2012). Using Semantic Differentials for an Evaluative View of the Search Engine as an Interactive System. In EuroHCIR (pp. 7-10) [http://ceur-ws.org/ Vol-909/paper2.pdf].

Kaparias, I., Bell, M. G. H., Miri, A., Chan, C., \& Mount, B. (2012). Analysing the perceptions of pedestrians and drivers to shared space. Transportation Research Part F: Traffic Psychology and Behaviour, 15(3), 297-310. [https://doi.org/10.1016/j.trf.2012.02.001].

Kyriakidis, M., Happee, R., \& de Winter, J. C. F. (2015). Public opinion on automated driving: Results of an international questionnaire among 5000 respondents. Transportation Research Part F: Traffic Psychology and Behaviour, 32, 127-140 [https://doi.org/10.1016/j. trf.2015.04.014].

Lange, J.-M., \& Victor, P. (2006). Didactique curriculaire et «éducation à... la santé, l'environnement et au développement durable»: quelles questions, quels repères? Didaskalia, (28), 85-100.

Lautrey, J. (2008). Les connaissances naïves chez l'enfant. In J. Lautrey, S. Rémi-Giraud, E. Sander, \& A. Tiberghien (Eds.), Les connaissances naïves (pp. 9-56). Paris: Armand Colin.

Lawrence, N. D. (2017). Living Together: Mind and Machine Intelligence. ArXiv:1705.07996 [Cs] [http:// arxiv.org/abs/1705.07996].

Legardez, A. (2006). Enseigner des questions socialement vives. Quelques points de repères. In A. Legardez \&
L. Simonneaux (Eds.), Lécole à l'épreuve de l'actualité. Enseigner les questions vives (pp. 19-32).

Legardez, A., \& Simonneaux, L. (2006). L'école à l'épreuve de l'actualité (vol. 110). Issy-les-Moulineaux: ESF.

Levinson, J., Askeland, J., Becker, J., Dolson, J., Held, D., Kammel, S., ... others. (2011). Towards fully autonomous driving: Systems and algorithms. In Intelligent Vehicles Symposium (IV), 2011 IEEE (pp. 163-168). IEEE [http://ieeexplore.ieee.org/xpls/abs_ all.jsp?arnumber=5940562].

Millard-Ball, A. (2016). Pedestrians, Autonomous Vehicles, and Cities. Journal of Planning Education and Research, 0739456X16675674.

Miller, D., Johns, M., Mok, B., Gowda, N., Sirkin, D., Lee, K., \& Ju, W. (2016). Behavioral Measurement of Trust in Automation: The Trust Fall. Proceedings of the Human Factors and Ergonomics Society Annual Meeting, 60(1), 1849-1853 [https://doi. org/10.1177/1541931213601422].

Osgood, C. E. (1964). Semantic differential technique in the comparative study of cultures. American Anthropologist, 66(3), 171-200.

Payre, W., Cestac, J., \& Delhomme, P. (2014). Intention to use a fully automated car: Attitudes and a priori acceptability. Transportation Research Part F: Traffic Psychology and Behaviour, 27, Part B, 252-263 [https:// doi.org/10.1016/j.trf.2014.04.009].

Simonneaux. (2003). Enseigner des savoirs "chauds": l'éducation biotechnologique entre science et valeurs. In J.-P. Astolfi (Ed.), Éducation et formation. Nouvelles questions, nouveaux métiers. Issy-les-Moulineaux: ESF.

Verberne, F. M. F., Ham, J., \& Midden, C. J. H. (2012). Trust in Smart Systems Sharing Driving Goals and Giving Information to Increase Trustworthiness and Acceptability of Smart Systems in Cars. Human Factors: The Journal of the Human Factors and Ergonomics Society, 54(5), 799-810 [https://doi. org/10.1177/0018720812443825].

Vosniadou, S., \& Brewer, W. F. (1992). Mental models of the earth: A study of conceptual change in childhood. Cognitive Psychology, 24(4), 535-585.

Vosniadou, S., \& Brewer, W. F. (1994). Mental Models of the Day/Night Cycle. Cognitive Science, 18(1), 123-183. [https://doi.org/10.1207/s15516709cog1801_4].

Yim, O., \& Ramdeen, K. T. (2015). Hierarchical cluster analysis: comparison of three linkage measures and application to psychological data. The Quantitative Methods for Psychology, 11(1), 8-21. 